\title{
IgG, but not IgM, mediates hyperacute rejection in hepatic xenografting
}

Schraa EO, Stockmann HBAC, Broekhuizen AJ., Scheringa M,
Schuurman HJ, Marquet RL, IJzermans JNM. IgG, but not IgM,
mediates hyperacute rejection in hepatic xenografting. Xenotransplantation 1999; 6: 110-116. (C) Munksgaard, Copenhagen

We reported previously that no classical features of hyperacute rejection (HAR) could be found in liver grafts in the guinea-pig (GP)-to-rat model and that recipients died shortly after transplantation of non-immunologic causes. Thus, the GP-to-rat model is not suitable for studying the mechanisms of discordant liver xenograft rejection. In the hamster to rat model, long-term survival of a liver graft is possible, but extremely low levels of xenoreactive natural antibodies are present. To mimic a discordant situation with pre-formed IgM and IgG antibodies, we sensitized rats 1 or 5 weeks before grafting. Specific anti-hamster IgM antibodies were found in recipients sensitized at week -1 but not week -5 . Anti-hamster IgG was present in all recipients, albeit considerably higher in animals sensitized 5 weeks before grafting. In these two models, we examined the mechanism of HAR of liver grafts and compared this with heart xenografts. Control heart and liver grafts were rejected 4 and 7 days after transplantation respectively. Liver grafts in recipients sensitized at week -5 showed venous congestion and bleeding after reperfusion, indicating HAR, however this was not observed after sensitization at week -1 . This surprising finding was confirmed by histology. Massive extravasation, edema, and acute liver cell degradation were noticed in grafts subjected to HAR. Liver grafts of recipients sensitized at week -1 showed only minimal changes. Heart grafts were rejected hyperacutely in both sensitization models. IgG antibodies could be detected on liver grafts in the group sensitized at week -5 but not in the group sensitized at week -1 . Minimal IgM depositions were found on liver grafts of animals sensitized 1 week before grafting. Rejected heart grafts from similar sensitization groups showed identical antibody depositions; only IgM depositions were massive. Complement depositions were found in all groups. These results indicate that $\mathrm{IgG}$, but not IgM, mediates HAR in hepatic xenografting. Such a predominance of IgG over IgM does not exist for heart grafts.

\author{
E. O. Schraa, ${ }^{1}$ H. B. A. C. \\ Stockmann, ${ }^{1}$ A. J. Broekhuizen, ${ }^{2}$ \\ M. Scheringa, ${ }^{1}$ H. J. Schuurman, ${ }^{2}$ \\ R. L. Marquet ${ }^{1}$ and \\ J. N. M. IJzermans ${ }^{1}$ \\ 'Department of Surgery, Erasmus University \\ Rotterdam, the Netherlands; ${ }^{2}$ Novartis Pharma \\ AG, Basle, Switzerland
}

Key words: liver - hamster - IgG antibody sensitization - xenotransplantation

Address reprint requests to

Dr E. O. Schraa, Laboratory for Experimental Surgery, Erasmus University Rotterdam, PO Box 1738, 3000 DR Rotterdam, The Netherlands.

Received 31 August 1998;

accepted 28 December 1998

\section{Introduction}

Transplantation of transgenic organs or tissue between widely disparate species as the ultimate solution for donor shortage shows promising results [1]. However, many aspects of rejection are still unknown, as evidenced by the muddle of immunosuppressive drugs that are needed to keep pig organs functional. Using small animals, the guinea-pig (GP)-to-rat rodent model provides an easy and fast way to study the basics of discordant transplantation. The recipient produces pre-formed xenoreactive natural antibodies (XNAs) against the GP, which are capable of rejecting heart grafts in a hyperacute manner [2]. XNAs are mainly IgM type [3], but some donorspecific IgG antibodies have been detected [4].

For allogeneic grafting, the relative insensitivity of liver grafts to antibody-mediated rejection has been described $[5,6]$. In a previous publication, we investigated whether this phenomenon 
also occurred in the GP-to-rat liver transplantation model [7]. We demonstrated that a 'rejected' GP liver was not characterized by the classical features of hyperacute rejection (HAR) [7]. However, recipients died within a few days, apparently because of non-immunological reasons. Therefore, no firm conclusions could be drawn regarding susceptibility of discordant liver graft toward antibody-mediated rejection [7]. Numerous studies have reported long-term survival of hamster grafts, indicating no interference of non-immunological problems in this model [810]. However, the rat has very low titers of preformed antibodies to hamster. To mimic a discordant situation with pre-formed IgM or IgG antibodies, we sensitized recipients with donor blood at 1 or 5 weeks before transplantation.

Hence, the aim of the current study was to analyze the mechanism of HAR of liver xenografts in the presence of pre-formed antibodies and to compare this with the rejection of heart xenografts.

\section{Materials and methods}

Animals

Female Syrian hamsters were used as donors and male Brown Norway rats as recipients. All animals were obtained from Harlan CPB (Austerlitz, The Netherlands). They were kept under controlled laboratory conditions and received food and tap water ad libitum. Hamsters weighing over $120 \mathrm{~g}$ and rats weighing between 250 and $300 \mathrm{~g}$ were used. The experimental protocols adhered to the rules laid down in the Dutch Animal Experimentation Act (1977) and the published Guidelines on the Protection of Experimental Animals by the Council of the EC (1986). The specific protocol was approved by the 'Committee on Animal Research' of the Erasmus University Rotterdam, The Netherlands.

\section{Liver transplantation}

Orthotopic liver transplantation (OLT) was performed according to a previously described method with some donor-related modifications $[7,11]$. The modifications were (1) donor pretreatment with $0.1 \mathrm{mg} / \mathrm{kg}$ atropine (Centrafarm Services, Etten-Leur, The Netherlands), and (2) the outside diameters of the Teflon cuffs were $2.1 \mathrm{~mm}$ and $1.79 \mathrm{~mm}$ for the IVC and PV respectively. Recipient death was taken as the end point of rejection.
Heart transplantation

Heterotopic abdominal heart transplantation (HTx) was performed as described by Ono and Lindsey [12]. Cessation of heartbeat as evidenced by abdominal palpation was taken as the end point of rejection.

\section{Sensitization}

Hamster blood was obtained by orbital punction. One milliliter of heparinized blood was injected i.v. into the penile vein of the recipient 1 or 5 weeks before organ grafting.

\section{Hemagglutination assay}

Total antibody and IgG levels were measured using a hemagglutination assay. Plasma samples, taken immediately before grafting, were serially diluted. A suspension of freshly prepared hamster erythrocytes $(4 \%)$ was added in equal amounts. After $1 \mathrm{~h}$ of incubation at $37^{\circ} \mathrm{C}$, the wells were screened for agglutination. The dilution at which agglutination still occurred was considered the hemagglutination titer. For IgG level measurements, plasma samples were treated with $0.5 \mathrm{mM}$ dithiothreitol for half an hour at $37^{\circ} \mathrm{C}$ to deplete IgM. Normal BN serum was taken as negative control. Estimations of IgM titers were calculated by subtracting IgG titers from total antibody titers.

\section{Histology and immunohistochemistry}

After rejection of the grafts, necropsy was performed. The heart graft or upper liver lobe was removed and processed for histology and immunohistochemistry. Paraffin sections were stained with hematoxylin-eosine and examined with conventional light microscopy. The slides were examined for extravasation, edema, vessel damage and infiltration. Changes were noted compared with naive grafts and scored from - (no changes) to +++ (severe changes)

For antibody, complement depositions and natural killer (NK) cell infiltration, immunohistochemistry on frozen sections was performed. Macrophages were identified on paraffin sections. FITC-labeled mouse anti-rat antibodies to demonstrate IgM, IgG1, IgG2a and IgG2b were used (1:10; PharMingen, San Diego CA, USA). $\mathrm{IgG} 2 \mathrm{c}$ and complement $\mathrm{C} 3$ were demonstrated by FITC-conjugated rabbit anti-sheep (1:100; Dako, Glostrup, Denmark) to sheep anti-rat antibodies (1:500; ANAWA Trading NA, Wangen Zurich, Switzerland). Complement factors $\mathrm{Clq}$ and $\mathrm{C} 9$ were stained by rabbit anti-rat $\operatorname{IgG}(1: 25,1: 600$, 
respectively, kindly provided by $\mathrm{Dr}$ B. P. Morgan) and secondary FITC-labeled swine anti-rabbit (Dako). Rat spleen was taken as positive control, whereas liver and heart from naive hamsters were used as negative controls. The slides were analyzed by fluorescence microscopy. Location of the depositions was noted and the fluorescence intensity was scored from - (no depositions) to +++ (massive depositions). A three-step indirect $\mathrm{Ni}-\mathrm{DAB}$ immunoperoxidase staining was performed on paraffin slides to demonstrate macrophage infiltration. Mouse anti-rat macrophage was used as primary antibody (ED1, 1:800; Serotec, Oxford, UK). Staining was performed with $\mathrm{Ni}-\mathrm{DAB}$ substrate after the application of rabbit anti-mouse $\mathrm{PO}$ and swine anti-rabbit PO antibodies (1:250; Dako). Applying the same protocol, NK cells were demonstrated on frozen sections using mouse anti-rat NK cells (NKR-P1, 1:400; Endogen, Woburn MA, USA). Conventional light microscopy was used to analyze the infiltration.

\section{Experimental design}

Heart and liver transplantations were carried out in different recipients. Sensitization with $1 \mathrm{ml}$ of hamster blood was performed 1 week before transplantation (1-week sensitized) in groups 2 and 5 and 5 weeks before transplantation (5-week sensitized) in groups 3 and 6 . Groups 2 and 3 received heart grafts $(\mathrm{n}=5$ for both); groups 5 and 6 obtained liver grafts $(n=7$ and $n=5$ respectively). Non-sensitized control groups were included for both heart (group 1, $\mathrm{n}=7$ ) and liver transplantations (group $4, n=8$ ). On day 0 the recipients obtained a heterotopic heart graft or orthotopic liver graft. Survival, as determined by abdominal palpation or death, was scored in minutes, hours or days, depending on the treatment. At necropsy the heart graft or upper liver lobe was removed and processed for histology and immunohistochemistry. Heart and liver grafts were semiquantitatively scored for type and quantity of antibody and histologic changes.

\section{Statistics}

Statistical evaluation of the survival data was carried out for both heart and liver grafts. In cases of differences in variances as tested by Levene's test, mathematical transformation of the survival data was carried out. This was carried out to reduce the influence of outlying values. 'One-way' analysis of variance (ANOVA) was performed on these data. If the ANOVA was significant on a $5 \%$ level, the Duncan's multiple comparison test or Games-Howell test was carried out for possible differences among the means. The tests were corrected for the fact that the comparisons were not statistically independent and for unequal group sizes.

Probability values lower than 0.05 were considered statistically significant. Survival of transplants with clear evidence of no rejection (liver enzymes, histology) was discarded from statistical evaluation. All computing was performed using the statistical software package SPSS for Windows, release 7.5.2.

\section{Results}

Histology

Specimens of the transplanted organs were obtained for histologic examination after killing the animals at the moment of rejection or death. Heart grafts rejected by non-sensitized rats showed vessel destruction, extravasation, edema and polymorphonuclear cell infiltrate, suggesting an antibody-mediated rejection. Also, the presence of mononuclear cells was noted. Groups 2 and 3 showed more severe extravasation of erythrocytes, fibrosis and edema. The overall architecture, including most vessels, was intact. In some cases, polymorphonuclear granulocytes were found.

Control liver grafts demonstrated polymorphonuclear infiltrate, destroyed vascular morphology with destroyed endothelial cell layers, edema, and a more predominant mononuclear cell infiltrate than heart grafts, consisting of lymphocytes, lymphoblasts, monocytes and macrophages. This indicates a mixture of cellular as well as antibody-mediated rejection. The infiltrate was situated around the portal areas.

Liver grafts of 1-week sensitized recipients showed extravasation, edema, signs of fibrosis and vascular congestion, but little vessel damage. Focally, mononuclear granulocytes were found. Specimens of liver grafts in group 6 revealed extensive tissue damage, extravasation and acute liver cell damage. Semiquantitative histology scores are listed in Table 1.

\section{Immunohistochemistry}

Frozen sections of heart and liver grafts were stained for IgM, IgG subtypes, complement $\mathrm{Clq}$, $\mathrm{C} 3$ and C9, and NK-cell infiltrates. Paraffin sections were used to stain for macrophages. All control stainings were positive on untreated rat spleen, whereas untreated hamster liver and heart sections were negative. 
Table 1. Histologic changes in heart and liver grafts, rejected by sensitized recipients

\begin{tabular}{llcccc}
\hline Group & \multicolumn{1}{c}{ Treatment } & Extravasation & Edema & Vessel damage & Infiltration \\
\hline 1 & HTX, non-sensitized & + & ++ & ++ & +++ \\
2 & HTX, 1-week-sensitized & ++ & ++ & + & \pm \\
3 & HTX, 5-week-sensitized & ++ & ++ & + & \pm \\
4 & OLT, non-sensitized & + & ++ & ++ & ++ \\
5 & OLT, 1-week-sensitized & + & + & + & \pm \\
6 & OLT, 5-week-sensitized & +++ & + & +++ & + \\
\hline
\end{tabular}

Scores varied from - (no changes) to +++ (severe changes) compared with naive heart/liver.

Control hearts showed major IgM depositions. Some IgG2a type antibodies and macrophages were noticed. Liver grafts from untreated rat recipients showed minor antibody depositions on cellular infiltrates or portal field, mainly being IgM. Macrophages, but not NK cells were deposited throughout the tissue.

Heart grafts from 1-week sensitized recipients demonstrated massive antibody depositions, whereas liver grafts demonstrated minor depositions. In groups 3 and 6 , moderate $\operatorname{IgG} 1$ depositions could be demonstrated. In addition, depositions of $\operatorname{IgG} 2 \mathrm{a}$ and $\mathrm{IgG} 2 \mathrm{~b}$ were detected on myocytes. For liver grafts depositions of $\operatorname{IgG} 2 \mathrm{~b}$ were found. Semiquantitative antibody deposition scores are listed in Table 2.

Liver grafts from untreated rat recipients showed some $\mathrm{Clq}$ depositions, but no $\mathrm{C} 3$ and $\mathrm{C} 9$, whereas all measured complement components could be detected on heart grafts. Sensitizing rats 1 week before transplantation resulted in moderate $\mathrm{C} 3$ depositions in both heart and liver grafts. In addition, positive Kupffer cells were found. C1q could only be detected in heart grafts, whereas little C9 was found in liver grafts. In 5-week sensitized recipients, massive $\mathrm{C} 3$ and moderate $\mathrm{C} 9$ depositions were demonstrated for heart grafts. Moderate C3 depositions and to a lesser extent $\mathrm{Clq}$ could be shown for liver grafts in group 6.

Table 2. Antibody depositions in rejected heart and liver grafts by sensitized recipients

\begin{tabular}{llccccc}
\hline Group & \multicolumn{1}{c}{ Treatment } & $\operatorname{lgM}$ & $\operatorname{lgG1}$ & $\operatorname{lgG} 2 \mathrm{a}$ & $\lg 2 \mathrm{~b}$ & $\lg \mathrm{g} 2 \mathrm{c}$ \\
\hline 1 & HTx, non-sensitized & +++ & - & + & - & - \\
2 & HTx, 1-week-sensitized & +++ & - & - & - & - \\
3 & HTx, 5-week-sensitized & - & ++ & + & + & - \\
4 & OLT, non-sensitized & + & \pm & \pm & \pm & \pm \\
5 & OLT, 1-week-sensitized & \pm & - & - & - & - \\
6 & OLT, 5-week-sensitized & - & ++ & \pm & + & - \\
\hline
\end{tabular}

Scores varied from - (no depositions) to +++ (massive depositions).

\section{Graft survival}

Heart and liver grafts showed a homogeneous reperfusion after releasing the clamps. After a few minutes, the heart grafts turned dark red. Liver grafts in group 6, but not group 5, were purple and showed swelling 2 min after reperfusion. Most of the recipients of liver grafts died showing the clinical signs of shock.

Graft survival times are shown in Table 3. A highly significant difference was demonstrated between the group means in the hearttransplanted groups $\left(P=0.000, \mathrm{~s}_{\text {res }}=35.730\right)$ and between the group means in the livertransplanted groups $\left(P=0.000, \mathrm{~s}_{\mathrm{res}}=0.567\right)$. Non-sensitized heart graft recipients showed prolonged survival compared with both other heart graft recipients $(P<0.001$, both $)$. No difference was found between 1-week-sensitized and 5-week-sensitized animals. The survival times of the non-sensitized group receiving liver grafts were significantly longer than those of animals in groups 5 and $6(P=0.000$, both). Survival times in groups 5 and 6 were not significantly different, but a trend was noticed $(P=0.081)$.

\section{$\lg \mathrm{M}$ and $\lg \mathrm{G}$ agglutination titers}

Hemagglutination tests revealed the presence of specific anti-hamster IgM antibodies in 1-weeksensitized animals on day 0 . The $\operatorname{IgM}$ titer was negligible in recipients sensitized 5 weeks before grafting. Anti-hamster IgG was present in all recipients, albeit considerably higher in recipients sensitized at week -5 . Normal BN serum revealed agglutinating titers of one-quarter or lower. No statistically significant correlation was found between pre-operative antibody titer and survival or histologic changes in either sensitization group.

Table 3. Survival times following 'pseudodiscordant' heart and liver transplantation. ${ }^{a}$

\begin{tabular}{|c|c|c|c|c|c|}
\hline Group & Surgery & Treatment & Survival times & & $\begin{array}{l}\text { Median } \\
\text { survival }\end{array}$ \\
\hline 1 & HTx & Non-sensitized & $3,4,4,4,4,4,4$ & days & 4 days \\
\hline 2 & HTx & 1-week-sensitized & $5,6,7,120,165$ & $\min$ & $7 \mathrm{~min}$ \\
\hline 3 & HTx & 5-week-sensitized & $3,3,3,9,10$ & $\min$ & $3 \min$ \\
\hline 4 & OLT & Non-sensitized & (3) $(4), 6,6,7,7,8,8$ & days & 7 days \\
\hline 5 & OLT & 1-week-sensitized & $1.5,1.5,2,2.5,3,21,25$ & h & $2.5 \mathrm{~h}$ \\
\hline 6 & OLT & 5-week-sensitized & $1,1,1.25,1.25,1.5$ & $\mathrm{~h}$ & $1.25 \mathrm{~h}$ \\
\hline
\end{tabular}




\section{Discussion}

The immunoprivileged position of liver grafts to antibody-mediated rejection has been a research subject for many years. Pre-formed antibodies in the circulation of patients before transplantation have been identified as the cause of HAR of allografts [13]. However, hyperacute antibodymediated rejection is not necessarily observed $[5,14]$. Liver allografts seem to be less susceptible to antibody-mediated rejection. The phenomenon of reduced susceptibility has been demonstrated in previous studies concerning experimental liver allotransplantation in (sensitized) recipients $[6,15]$. In discordant liver grafting, we and others were unable to resolve this question, because the recipients died from nonimmunological problems $[7,16]$. For that reason, we performed the current experiments. Similarly, as described by others, we found that nonsensitized controls rejected hearts significantly earlier than liver grafts $[10,17]$. Histologically, control heart grafts showed a prominent vascular rejection process, whereas rejected liver grafts (group 4) revealed a more predominant cellular rejection with mononuclear cell infiltrates. Moreover, antibody depositions of the IgM type were more pronounced on heart grafts than liver grafts. These findings indicate that in the untreated concordant hamster to rat combination the liver might be less affected by IgM.

It can be argued that liver grafts are less immunogenic than heart grafts, resulting in a diminished and sluggish antibody response resulting in less damage. However, this is not very plausible, because anti-donor antibody levels peak around days 5-7 for both liver and heart transplantation [18-20]. Moreover, Murase et al. [21] reported that cytotoxic antibody titers after xenogeneic liver grafting are 10 times higher than after heart grafting. Infusion of hamster hepatocytes or non-parenchymal liver cells was able to induce high cytotoxic antibody titer within 1 week, and splenic response to liver grafts is even higher than to cardiac grafts [22,23]. This indicates that liver grafts may be at least as immunogenic as heart grafts.

Another plausible explanation is that the liver graft protects itself, because the source of complement and the target organ are the same, leading to prolonged survival [24]. This is in accordance with the time required to transform the proteins to donor type profile [7,25]. Nevertheless, residual recipient complement components and neosynthesis, by macrophages, monocytes, and fibroblasts, are still able to cause lysis [24].
In normal $\mathrm{BN}$ serum, hemagglutinating antibodies were almost non-detectable. In the discordant situation, grafted organs encounter high titers of pre-formed antibodies. Immunogenicity is therefore of less relevance than the affinity of antibodies for the different tissues. To mimic the pre-formed antibody situation, we sensitized rat recipients with hamster antigen in order to evoke an antibody response. This sensitization, 1 or 5 weeks before transplantation, resulted in graft survival of minutes to hours for both liver and heart grafts. The differences found between liver and heart graft survival in the sensitized situation may be explained by the difference in survival readout for the grafts. It is known that rats can live for several hours after total hepatectomy with just a portal caval shunt [7].

At necropsy, massive antibody depositions could be detected in heart grafts from 1-week sensitized recipients. Extravasation of erythrocytes, edema, fibrosis and vascular congestion, suggesting complement-mediated endothelial damage, indicate an ongoing HAR. Liver grafts from recipients sensitized 1 week before transplantation, however, showed an overall intact morphology and only marginal signs of HAR. This corresponds to their phenotypic appearance after grafting. Yet, classical signs of HAR were noted in liver grafts from 5-week sensitized animals: acute liver cell degradation, extensive tissue damage and extravasation of erythrocytes. Purple recoloration and swelling were noticed after reperfusion. Immunohistochemistry showed IgG depositions, whereas no graft depositions could be found in rats sensitized at week -1 .

Circulating IgM, present 1 week after sensitization, is likely to be deposited on the heart as well as liver grafts. However, the results indicate that liver grafts, in contrast to heart grafts, seem to be less affected by IgM as seen by immunohistochemisty and complement depositions. One possibility is that the liver is releasing blocking agents, preventing antibody deposition, which has been hypothesized by Kamada et al. [26] Another explanation is that graft size difference gives rise to difference in deposition density and therefore rejection. However, a more likely possibility is that Kupffer cells absorb large amounts of lymphocytotoxic antibodies [27,28]. Moreover, Crafa et al. [29] reported that Kupffer cell activation led to significant reduced circulation of anti-donor antibodies and a more intense IgM uptake after discordant liver grafting as demonstrated by immunohistochemistry. In the present study, however, liver grafts showed minor IgM depositions. Other authors have reported similar findings. Tuso et al. [30] demonstrated 
that xenogeneic extracorporeal liver perfusion resulted in minimal immunohistochemical evidence of binding of human xenoantibodies. Nevertheless, reduced antibody binding to other organs was found after xenogeneic liver perfusion [30]. Even prolonged heart xenograft survival has been reported after preceding xenogeneic liver transplantation [31]. This indicates that liver grafts, unlike heart grafts, are less susceptible to antibody-mediated damage, probably because of absorption or non-binding of antibodies, possibly IgM.

Five weeks after sensitization, mainly IgG-type antibodies prevail. Histology of rejected livers showed a severe HAR with IgG and complement deposition, which were not detected in the grafts after recipient sensitization at week -1 . It seems therefore that IgG, specifically some IgG subtypes, may be responsible for the rejection process in liver grafts. In addition, several authors found that in experimental allogeneic liver transplantation after recipient sensitization graft failure was dependent on the antibody class being $\operatorname{IgG}[32,33]$. Moreover, $\operatorname{IgG}$, in contrast to IgM, seems to be the most dangerous in clinical allotransplantation across positive cross-matches $[34,35]$. The minor damage seen in liver grafts of 1-week sensitization recipients might be caused by the already formed, relatively low titers of IgG.

This could also explain why in discordant grafting no signs of HAR were seen, because most XNAs are of the IgM type $[3,4,7,36]$.

In conclusion, this study suggests a more dominant role for IgG over IgM antibodies in the rejection of liver xenografts. Such predominance does not exist for heart grafts.

\section{References}

1. Bhatti FNK, Schmoeckel M, Zaidi A, et al. Three month survival of hDAF transgenic pig hearts transplanted into primates. Abstracts of the Transplantation Society, XVII World Congress, Transplant 981998 (Abstract 138).

2. Leventhal JR, Flores HC, Gruber SA, et al. Evidence that 15-deoxyspergualin inhibits natural antibody production but fails to prevent hyperacute rejection in a discordant xenograft model. Transplantation 1992; 54: 26.

3. Gambiez L, Salame E, Chereau C, et al. The role of natural IgM in the hyperacute rejection of discordant heart xenografts. Transplantation 1992; 54: 577.

4. Leventhal J, Figueroa J, Flores H, Platt Jl, Bach FH. Measurement of natural antibody in a discordant xenograft model. Transplant Proc 1992; 24: 455.

5. Iwatsuki S, Rabin BS, Shaw BW Jr, Starzl TE. Liver transplantation against $\mathrm{T}$ cell-positive warm crossmatches. Transplant Proc 1984; 16: 1427.
6. Kamada N, Shinomiya T. Serology of liver transplantation in the rat. I. Alloantibody responses and evidence for tolerance in a nonrejector combination. Transplantation 1986; $42: 7$.

7. Schraa EO, Schotman SN, Scheringa M, Daha MR, Marquet RLI, Jzermans Jnm. Discordant liver transplantation does not lead to classical hyperacute rejection. Xenotransplantation 1996; 3: 321.

8. Steinbruchel DA, Nielsen B, Kemp E. Treatment of hamster heart to rat xenotransplantation. Transpl Immunol 1994; 2: 3.

9. Boumman E, de Bruin RW, Jeekel J, Marquet Rl. Recipient pretreatment permits long-term xenograft survival on a relatively low dose cyclosporine maintenance therapy. Transplant Proc 1992; 24: 519.

10. Celli S, Valdivia LA, Fung JJ, et al. Long-term survival of heart and liver xenografts with splenectomy and FK 506. Transplant Proc 1993; 25: 647.

11. Kamada N, Calne RY. A surgical experience with five hundred thirty liver transplants in the rat. Surgery 1983; 93: 64 .

12. ONO K, LindSey ES. Improved technique of heart transplantation in rats. J Thorac Cardiovasc Surg 1969; 57: 225.

13. Kissmeyer-Nielsen F, Olsen S, Petersen VP, Fueldborg O. Hyperacute rejection of kidney allografts, associated with pre-existing humoral antibodies against donor cells. Lancet 1966; 2: 662.

14. Starzl TE, Ishikawa M, Putnam CW, et al. Progress in and deterrents to orthotopic liver transplantation, with special reference to survival, resistance to hyperacute rejection, and biliary duct reconstruction (Review). Transplant Proc 1974; 6: 129.

15. Wang C, Sun J, Li L, Wang L, Dolan P, Sheil AG. Conversion of pancreas allograft rejection to acceptance by liver transplantation. Transplantation 1998; 65: 188.

16. Settaf A, Meriggi F, Van de stadt J, et al. Delayed rejection of liver xenografts compared to heart xenografts in the rat. Transplant Proc 1987; 19: 1155.

17. Murase N, Starzl TE, Demetris AJ, et al. Hamster-torat heart and liver xenotransplantation with FK506 plus antiproliferative drugs. Transplantation 1993; 55: 701.

18. Valdivia LA, Monden M, Gotoh M, et al. Prolonged survival of hamster-to-rat liver xenografts using splenectomy and cyclosporine administration. Transplantation 1987; 44: 759.

19. van den Bogaerde J, Hassan R, White DG. An analysis of concordant xenografting. Transplant Proc 1992; 24 : 513.

20. Lin Y, Vandeputte M, Waer M Factors involved in rejection of concordant xenografts in complementdeficient rats. Transplantation 1997; 63: 1705.

21. Murase N, Demetris AJ, Tanabe M, et al. Effect of FK 506 and antiproliferative agents for heart and liver xenotransplantation from hamster to rat. Transplant Proc 1993; 25: 425.

22. Tsugita M, Valdivia LA, Rao AS, et al. Tacrolimus pretreatment attenuates preexisting xenospecific immunity and abrogates hyperacute rejection in a presensitized hamster to rat liver transplant model. Transplantation 1996; 61: 1730.

23. Langer A, Valdivia LA, Murase N, et al. Humoral and cellular immunopathology of hepatic and cardiac hamster-into-rat xenograft rejection. Marked stimulation of $\mathrm{IgM}++$ bright/IgD+dull splenic B cells. Am J Pathol 1993; 143: 85.

24. Valdivia LA, Fung JJ, Demetris AJ, et al. Donor species 


\section{Schraa et al.}

complement after liver xenotransplantation. The mechanism of protection from hyperacute rejection. Transplantation 1994; 57: 918.

25. Valdivia LA, Lewis JH, Celli S, et al. Hamster coagulation and serum proteins in rat recipients of hamster xenografts. Transplantation 1993; 56: 489.

26. Kamada N, Davies HS, Roser B. Reversal of transplantation immunity by liver grafting. Nature 1981; 292: 840.

27. Amorosa L, Gugenheim J, Saint-Paul MC, Benzaken S, Mouiel J. Prolongation of heart xenograft survival after liver hemoperfusion. Transplant Proc 1990; 22: 2002.

28. Astarcioglu I, Gugenheim J, Crafa F, Saint Paul MC, ReYNES M. Hyperacute rejection of liver allografts in sensitized rats: role of nonparenchymal liver cells. J Surg Res 1995; 58: 182.

29. Crafa F, Gugenheim J, Saint-Paul MC, Lapalus F, Damais A, Mouiel J. Role of nonparenchymal liver cells in guinea pig to rat hepatic xenotransplantation. Eur Surg Res 1993; 25: 303.

30. Tuso PJ, Cramer DV, Yasunaga C, Cosenza CA, Wu GD, Maкоwкa L. Removal of natural human xenoantibodies to pig vascular endothelium by perfusion of blood through pig kidneys and livers. Transplantation 1993; 55: 1375.

31. Valdivia LA, Demetris AJ, Fung JJ, et al. Hamster-torat liver xenografts protect extrahepatic organs from rejection. Transplant Proc 1993; 25: 414.

32. Furuya T, Murase N, Nakamura K, et al. Preformed lymphocytotoxic antibodies: the effects of class, titer and specificity on liver vs. heart allografts. Hepatology 1992; 16: 1415.

33. Nakamura K, Murase N, Becich MJ, et al. Liver allograft rejection in sensitized recipients. Observations in a clinically relevant small animal model. Am J Pathol 1993; 142: 1383.

34. Demetris AJ, Nakamura K, Yagihashi A, et al. A clinicopathological study of human liver allograft recipients harboring preformed IgG lymphocytotoxic antibodies. Hepatology 1992; 16: 671.

35. Iwaki Y, Lau M, Terasaki PI. Successful transplants across $\mathrm{T}$ warm-positive crossmatches due to IgM antibodies. Clin Transplant 1988; 2: 81.

36. Platt JL, Lindman BJ, Geller RL, et al. The role of natural antibodies in the activation of xenogenic endothelial cells. Transplantation 1991; 52: 1037. 